\title{
Características de las plantas medicinales comercializadas en diferentes mercados de Lima Metropolitana y sus efectos sobre el medio ambiente y la salud pública
}

\author{
Jesús Silva Alarcón* 1,a; Jorge Cabrera Meléndez ${ }^{1, b}$; Omar V. Trujillo Villarroel 1,c; Ivonne F. Reyes-Mandujano ${ }^{1, d}$
} RESUMEN

Objetivo: Evaluar las características de las plantas medicinales comercializadas en diferentes mercados de Lima Metropolitana y sus efectos sobre el medio ambiente y la salud pública.

Materiales y métodos: Se realizó un estudio descriptivo en mercados populares de Lima. Los datos se obtuvieron a través de entrevistas semiestructuradas y observación no participante. Las variables consideradas fueron los nombres comunes, el origen, la forma de venta, la parte comercializada, el uso reportado, la estacionalidad y las especies más demandadas. Se consultó bibliografía especializada para determinar el origen de la especie y la categorización de especies amenazadas de flora silvestre para el estado de amenaza.

Resultados: Se registraron 219 especímenes de plantas medicinales; se identificaron 183 que correspondían a 65 familias botánicas, principalmente, Asteraceae (37) y Lamiaceae (17), algunas de ellas en peligro de extinción.

Conclusiones: Las plantas provienen, mayormente, de las tierras altas peruanas y se comercializan en forma de "atados". Una misma especie puede ser ofrecida para el tratamiento de varios problemas de salud. La demanda aumenta en invierno ( >85 especies). Las especies que se venden con mayor frecuencia son manzanilla, orégano, cedrón, toronjil, yerbaluisa, hinojo, muña, sábila o aloe, llantén y coca. Del total de especies identificadas, el $10 \%$ está en peligro de extinción.

Palabras clave: Comercialización; Plantas medicinales; Medicina tradicional; Etnobotánica (Fuente: DeCS: BIREME).

\section{Characteristics of medicinal plants traded in different popular markets of Lima Metropolitan Area and their effects on the environment and public health}

\section{ABSTRACT}

Objective: To evaluate the characteristics of medicinal plants traded in different popular markets of Lima Metropolitan Area and their effects on the environment and public health.

Materials and methods: A descriptive study was carried out in popular markets of Lima. Data was collected through semi-structured interviews and non-participant observation. The variables to be considered were common names, origin, retailing/wholesaling, part of the plant to be traded, reported use, seasonality and most demanded species. Specialized bibliography was reviewed to determine the origin of the species and their threat categorization for endangered wild flora species.

Results: Two hundred ninteeen (219) species of medicinal plants were collected, out of which 183 were taxonomically identified and belonged to 65 botanical families, mostly Asteraceae (37) and Lamiaceae (17), some of them in danger of extinction.

Conclusions: The plants mainly come from the Peruvian highlands and are marketed in "bundles". A same species whose demand is increased in winter ( $>85$ species) may be offered for the treatment of several health problems. The most frequently sold species are chamomile, oregano, cedron, melissa or lemon balm, lemon grass, fennel, muña (Minthostachys mollis), aloe, broadleaf plantain and coca. Out of the total number of species identified, $10 \%$ is endangered.

Keywords: Commerce; Plants, medicinal; Medicine, traditional; Ethnobotany (Source: MeSH NLM).

1. Instituto Nacional de Salud. Centro Nacional de Salud Intercultural. Lima, Perú.

a. Ingeniero Agrónomo.

b. Biólogo, con meción en Botánica.

c. Magister en Epidemiología, Médico.

d. Magister en Biología Molecular, Biólogo.

* Autor corresponsal 


\section{INTRODUCCIÓN}

Las plantas medicinales representan la fuente más importante de recursos para el tratamiento de diferentes enfermedades. El uso indebido de estos recursos naturales tiene un impacto negativo en el medio ambiente, los ingresos de los proveedores de materias primas, las compañías farmacéuticas y, principalmente, en los usuarios tradicionales ${ }^{(1)}$.

El uso de plantas medicinales se ha extendido debido a su accesibilidad, bajo costo y efectos adversos atribuidos a las drogas sintéticas (2). Sin embargo, esta demanda afecta su disponibilidad y la salud de los consumidores tradicionales ${ }^{(3)}$. En el Perú se pueden reconocer dos tipos de comercialización de plantas medicinales; el primero incluye fitofármacos, productos naturales y derivados, que están regulados por el Ministerio de Salud; y el segundo se caracteriza por la desorganización, inseguridad y desconocimiento de la efectividad y calidad de las plantas que se venden frescas o secas en mercados populares, sin regulación institucional.

Existen varios estudios sobre plantas medicinales en mercados populares que reportan que los vendedores ofrecen a los clientes información incorrecta sobre el uso de las plantas medicinales (4) y condiciones de higiene insatisfactorias ${ }^{(5)}$; así también, el mismo nombre común se usa frecuentemente para identificar diferentes especies botánicas ${ }^{(6)}$. A pesar de ello, los mercados populares representan lugares estratégicos para la investigación etnobotánica (7), especialmente, los relacionados con el uso tradicional de las plantas medicinales ${ }^{(8-11)}$ y la biodiversidad ${ }^{(2,11,12)}$.

En Lima, capital y principal ciudad el Perú, existen muchos mercados populares visitados masivamente por público que adquiere plantas medicinales en su estado natural y en preparaciones muy simples y cuya dinámica no ha sido investigada, por lo que el objetivo de este trabajo fue evaluar las características de las plantas medicinales comercializadas en diferentes mercados de Lima Metropolitana y sus efectos sobre el medio ambiente y la salud pública, con el propósito de identificar especies comercializadas, usos tradicionales reportados, condiciones de distribución, origen, diversidad de la flora medicinal y variedades de mayor demanda.

\section{MATERIALES Y MÉTODOS}

Diseño y población

El tipo de estudio fue descriptivo. Los mercados se seleccionaron mediante la observación mensual durante el año 2014, y en base al número de habitantes locales y a la existencia de vendedores de plantas medicinales.

La información se recopiló mediante entrevistas anónimas semiestructuradas en el momento de la recolección de muestras de plantas medicinales y mediante la observación no participante. Para determinar el origen de las muestras se consultó el Catálogo de angiospermas y gimnospermas del Perú (13). Las determinaciones taxonómicas se realizaron en el Herbario del Centro Nacional de Salud Intercultural (CENSI) del Instituto Nacional de Salud del Perú, por un biólogo especialista en botánica, que comparó las muestras herborizadas con las existentes en la colección del mencionado herbario y analizó la bibliografía especializada ${ }^{(13-15)}$. La clasificación botánica se basó en la propuesta de la APGIII (2009) ${ }^{(15)}$. Las especies amenazadas se identificaron en la Categorización de especies de flora silvestre en peligro de extinción ${ }^{(14)}$. Los 36 vendedores entrevistados fueron seleccionados con base en la diversidad de plantas medicinales que ofrecían al público, el grado de experiencia (más de 10 años) y la voluntad de participar en la entrevista

\section{Variables del estudio}

Las variables consideradas fueron especies, origen, hábito de la planta, presentación del producto, parte de la planta, uso tradicional reportado por el vendedor, estacionalidad, número y estado de conservación (amenazada o no).

\section{Análisis estadístico}

El análisis estadístico incluyó el cálculo de las frecuencias absolutas y relativas de las variables, para lo que se utilizó el programa Microsoft Excel 2010.

\section{Consideraciones éticas}

Este estudio fue aprobado por el Comité de Ética del Instituto Nacional de Salud en Lima-Perú (RD No. 992 -2013-DG-OGITT-OPE/INS). Antes de la entrevista, se obtuvo el consentimiento verbal de los entrevistados. Los datos fueron recogidos de forma anónima.

\section{RESULTADOS}

Los mercados en los que se realizó la investigación aparecen en la la tabla 1. En ellos se encontró una cantidad adecuada de vendedores de plantas medicinales y un público que busca este producto. 
Tabla 1. Distribución de la muestra de mercados, según distritos

\begin{tabular}{|ll|}
\hline \multicolumn{1}{|c}{ Distritos } & \multicolumn{1}{c}{ Mercados } \\
\hline Puente Piedra & Señor de Huamantanga \\
\hline Comas & Complejo Comercial Unicachi - Retablo, Megamercado Unicachi - Pro \\
\hline Los Olivos & Conzac \\
\hline San Martín de Porres & San Martín de Porres - Condevilla \\
\hline Cercado & Ramón Castilla (Central) \\
\hline La Victoria & Aviación (La Parada) \\
\hline Ate & La Arenera - Huaycán, Ceres \\
\hline San Juan de Lurigancho & Valle Sagrado, Sarita Colonia \\
\hline Pachacamac & Virgen del Carmen de Los Huertos de Manchay \\
\hline Villa El Salvador & Mayorista Unicachi - Lima Sur \\
San Juan de Miraflores & San Juan de Miraflores \\
\hline
\end{tabular}

Se colectaron un total de 219 especies de plantas medicinales y se determinaron taxonómicamente 183, que correspondieron a 65 familias botánicas entre las que destacan las Asteraceae, Lamiaceae y Fabaceae (Tabla $2)$. Como se esperaba, se observaron diferencias en el número de especies entre los mercados. La Parada, Señor de Huamantanga y Ceres aportaron más del $70 \%$ del total de especímenes. El comercio al por mayor de plantas medicinales frescas se realiza en el distrito de La Victoria, de 1:00 a 8:00 a. m., sobre todo los martes y viernes de cada semana.

Las plantas provienen de diferentes lugares del país, especialmente, de Huaraz, Tarma, Huancayo, Huánuco, Cerro de Pasco, Huancavelica y las partes altas de Lima. En general, del total de plantas medicinales vendidas en los mercados, 73,8 \% (135) de ellas vienen de la sierra; 13,7 $\%$ (25), de la amazonía, y 12,6 \% (23), de la costa. Por otro lado, se observa que el $66,1 \%$ (121) son nativas, mientras que las especies introducidas alcanzan el 33,9 \% (62). Según la forma de obtención, el 50,3 \% (92) son silvestres, e incluyen algunos naturalizados, y el 49,7\% (91) se cultivan. Predominan las hierbas, seguidas de arbustos y árboles; las lianas y hemiparásitos son las menos diversas. El 65,6 $\%$ de las plantas que se comercializan son frescas, el 21,3 $\%$ incluye frescas y secas, y solo el $13,1 \%$ son secas. Las plantas frescas provienen, principalmente, de los andes y la costa.

Con respecto a la comercialización de las partes de las plantas, $46,4 \%$ corresponde a la parte aérea; $14,2 \%$, a toda la planta; $11,5 \%$, a los frutos; $10,4 \%$, a las hojas; $6,6 \%$, a las partes subterráneas (raíz y rizoma); 5,5\%, a las flores, y $4,4 \%$, a semillas. En cuanto al uso, se observó que la misma especie se comercializa para el tratamiento de varios problemas de salud (Tabla 2).

Tabla 2. Especies medicinales comercializadas por categoría de uso reportado

\begin{tabular}{|c|c|c|c|}
\hline Categoría-Sistema & Especies & $\mathbf{N}$ & $\%$ \\
\hline $\begin{array}{c}\text { Sistema } \\
\text { gastrointestinal } \\
\text { y enfermedades } \\
\text { hepáticas }\end{array}$ & $\begin{array}{l}\text { Cichorium intybus L., Artemisia absinthium L., Cynara scolymus } \\
\text { L., Tagetes filifolia Lag., Niphogeton scabra (H. Wolff) J.F. Macbr., } \\
\text { Momordica balsamina L., Rorippa nasturtium-aquaticum (L.) Hayek, } \\
\text { Peumus boldus Molina, Baccharis genistelloides (Lam.) Pers., Aloysia } \\
\text { citriodora Paláu, Phyllanthus niruri L., Adiantum poiretii Wikstr... }\end{array}$ & 49 & 36,6 \\
\hline $\begin{array}{l}\text { Sistema genitourinario } \\
\text { de hombres y mujeres } \\
\text { (niveles excretor y } \\
\text { reproductor) }\end{array}$ & $\begin{array}{l}\text { Acaulimalva engleriana (Ulbr.) Krapov., Niphidium sp., Phyllanthus } \\
\text { urinaria L., Mutisia acuminata Ruiz \& Pav., Equisetum giganteum L., } \\
\text { Cynodon dactylon (L.) Pers., Prunus serotina Ehrh., Chuquiraga spinosa } \\
\text { Less., Lupinus sp., Xanthium spinosum L., Zea mays L., Malva arborea } \\
\text { (L.) Webb \& Berthel., Alcea rosea L., Desmodium molliculum (Kunth) } \\
\text { DC., Tropaeolum tuberosum Ruiz \& Pav., Jungia paniculata (DC.)A.Gray, } \\
\text { Urtica flabellata Kunth, Tessaria integrifolia Ruiz \& Pav., Erythrina edulis } \\
\text { Triana ex Micheli, Bejaria aestuans Mutis ex L., Echinopsis pachanoi } \\
\text { (Britton \& Rose) Friedrich \& G.D. Rowley, Mauritia flexuosa L. f., ... }\end{array}$ & 56 & 36,6 \\
\hline
\end{tabular}


Características de las plantas medicinales comercializadas en diferentes mercados de Lima Metropolitana y sus efectos sobre el medio ambiente y la salud pública

\begin{tabular}{|c|c|c|c|}
\hline Categoría-Sistema & Especies & $\mathbf{N}$ & $\%$ \\
\hline $\begin{array}{l}\text { Sistema } \\
\text { cardiovascular y } \\
\text { circulatorio }\end{array}$ & $\begin{array}{l}\text { Allium sativum L., Medicago sativa L., Luma chequen (Feuillée ex } \\
\text { Molina) A. Gray, Cyphomandra betacea (Cav.) Sendtn., Cyclanthera } \\
\text { brachybotrys (Poepp. \& Endl.) Cogn., Schkuhria pinnata (Lam.) Kuntze } \\
\text { ex Thell., Salvia hispanica L., Solanum sessiliflorum Dunal, Rumex sp., } \\
\text { Gentianella sp, Puya fastuosa Mez, Ormosia sp.,..... }\end{array}$ & 50 & 27,3 \\
\hline $\begin{array}{l}\text { Enfermedades } \\
\text { respiratorias }\end{array}$ & $\begin{array}{l}\text { Pimpinella anisum L., Aristeguietia ballii (Oliv.) R.M. King \& H. Rob., } \\
\text { Borago officinalis L., Clerodendrum chinense (Osbeck) Mabb., Myrciaria } \\
\text { dubia (Kunth) McVaugh, Erythroxylum coca Lam., Perezia multiflora } \\
\text { (Humb. \& Bonpl.) Less. Eucalyptus globulus Labill. Punica granatum L... }\end{array}$ & 49 & 26,8 \\
\hline $\begin{array}{l}\text { Sistema } \\
\text { Musculoesquelético } \\
\text { (trastornos y } \\
\text { traumas asociados } \\
\text { con articulaciones, } \\
\text { músculos o hueso) }\end{array}$ & $\begin{array}{l}\text { Achyrocline alata (Kunth) DC., Arracacia xanthorrhiza Bancroft., } \\
\text { Baccharis sp., Tagetes elliptica Sm., Grindelia glutinosa (Cav.) Mart., } \\
\text { Oenothera rosea L'Hér. ex Aiton, Zingiber officinale Rosc., Schinus } \\
\text { molle L., Urtica sp., Jatropha gossypiifolia L., Sambucus peruviana } \\
\text { Kunth, Ligaria cuneifolia (Ruiz \& Pav.) Tiegh., Bejaria sp., Coreopsis } \\
\text { fasciculata Wedd., Azorella biloba (Schltdl.) Wedd., Medicago sativa L., } \\
\text { Jungia paniculata (DC.) A. Gray, Urtica urens L., Erythrina edulis Triana } \\
\text { ex Micheli, Chenopodium quinoa Willd., Spartium junceum L., Verbena } \\
\text { litoralis Kunth, Theobroma cacao L., Peperomia inaequalifolia Ruiz \& } \\
\text { Pav., Petiveria alliacea L., Chenopodium sp., Gaiadendron punctatum } \\
\text { (Ruiz \& Pav.) G.Don, Urtica flabellata Kunth... }\end{array}$ & 28 & 15,3 \\
\hline $\begin{array}{l}\text { Enfermedades } \\
\text { dermatológicas } \\
\text { (piel o mucosas) }\end{array}$ & $\begin{array}{l}\text { Calendula officinalis L., Piper aduncum L., Piper acutifolium Ruiz \& Pav., } \\
\text { Gamochaeta americana (Mill.) Wedd., Chenopodium quinoa Willd., } \\
\text { Aloe vera (L.) Burm.f., Gaiadendron punctatum (Ruiz \& Pav.) G. Don, } \\
\text { Nicotiana tabacum L., Solanum mammosum L., Mauritia flexuosa L. f., } \\
\text { Achyrocline alata (Kunth) DC., Baccharis genistelloides (Lam.) Pers., } \\
\text { Rumex sp., Cestrum auriculatum L'Hér., Plantago major L., Alcea rosea } \\
\text { L., Juglans neotropica Diels, Jatropha gossypiifolia L., Chenopodium } \\
\text { sp.,... }\end{array}$ & 28 & 15,3 \\
\hline $\begin{array}{l}\text { Sistema } \\
\text { nervioso }\end{array}$ & $\begin{array}{l}\text { Matthiola incana (L.) W.T. Aiton, Tagetes erecta L., Lavandula sp., } \\
\text { Matricaria chamomilla L., Passiflora edulis Sims., Citrus sinensis (L.) } \\
\text { Osbeck, Viola tricolor L., Chenopodium sp., Valeriana sp., Pimpinella } \\
\text { anisum L., ... }\end{array}$ & 19 & 10,4 \\
\hline $\begin{array}{l}\text { Enfermedades } \\
\text { endocrinas, } \\
\text { nutricionales y } \\
\text { metabólicas (diabetes) }\end{array}$ & $\begin{array}{l}\text { Physalis peruviana L., Cyclanthera pedata (L.) Schrader, Gentianella } \\
\text { thyrsoidea (Hook.) Fabris, Argyrochosma nivea (Poir.) Windham, } \\
\text { Cheilanthes pruinata Kaulf., Stevia rebaudiana (Bertoni) Bertoni, Ficus } \\
\text { carica L., Morus alba L., Geranium sp., Hylocereus trigonus (Haw.) Saff. } \\
\text { Smallanthus sonchifolius (Poepp. \& Engler) H. Robinson... }\end{array}$ & 18 & 9,8 \\
\hline $\begin{array}{l}\text { Síndromes culturales } \\
\text { (colerina, susto, mal } \\
\text { aire, etc.) }\end{array}$ & $\begin{array}{l}\text { Sonchus oleraceus L., Dianthus caryophyllus L., Peperomia } \\
\text { inaequalifolia Ruiz \& Pav., Sanguisorba minor Scop., Gentianella sp., } \\
\text { Laccopetalum giganteum (Wedd.) Ulbr., Aeonium sp., Ruta chalepensis } \\
\text { L., Matricaria chamomilla L., Viola tricolor L., Senecio richii A.Gray, } \\
\text { Tanacetum parthenium (L.) Sch.Bip., Valeriana sp., Peperomia galioides } \\
\text { Kunth, Melissa officinalis L. }\end{array}$ & 15 & 8,2 \\
\hline $\begin{array}{c}\text { Neoplasias } \\
\text { (cáncer y tumores) }\end{array}$ & $\begin{array}{l}\text { Bixa orellana L., Cynanchum tarmense Schltr., Annona muricata L., } \\
\text { Stachys arvensis (L.) L., Symphytum officinale L., Curcuma longa L., } \\
\text { Allium sativum L., ... }\end{array}$ & 14 & 7,7 \\
\hline $\begin{array}{c}\text { Órganos de los } \\
\text { sentidos (vista y oído) }\end{array}$ & $\begin{array}{l}\text { Senecio richii A.Gray, Physalis peruviana L., Achyrocline alata (Kunth) } \\
\text { DC., Peperomia inaequalifolia Ruiz \& Pav., Matricaria chamomilla L., } \\
\text { Ruta chalepensis L. }\end{array}$ & 6 & 3,3 \\
\hline $\begin{array}{l}\text { Otros (fiebre, cefalea, } \\
\text { inflamaciones } \\
\text { internas) }\end{array}$ & $\begin{array}{l}\text { Theobroma cacao L., Hordeum vulgare L., Solanum americanum Mill., } \\
\text { Cestrum auriculatum L'Hér., Plantago major L., Lepidium meyenii } \\
\text { Walpers., Ageratina sp., Petiveria alliacea L., Tanacetum parthenium (L.) } \\
\text { Sch.Bip., Melilotus indicus (L.) All. }\end{array}$ & 26 & 14,4 \\
\hline
\end{tabular}


La cantidad de plantas medicinales varía de acuerdo a la temporada, en invierno se registró el mayor número de especímenes. En general, la presentación del producto (planta medicinal) es el "atado" o "paquete". Las especies más vendidas fueron Matricaria chamomilla L. "manzanilla", Origanum vulgare L. "orégano", Aloysia citriodora Paláu "cedrón", Melissa officinalis L. "toronjil", Cymbopogon citratus (DC.) Stapf " yerbaluisa ", Foeniculum vulgare Mill. "hinojo", Minthostachys mollis (Kunth) Griseb. "muña", Aloe vera (L.) Burm.f. "sábila o aloe", Plantago major L. "llantén" y Erythroxylum coca Lam. "coca".

El $10 \%$ de las especies identificadas en nuestro estudio presentó algún grado de amenaza de extinción. De acuerdo a la regulación actual en el país ${ }^{(14)}$, se reportan en peligro crítico ( 3 especies), en peligro ( 2 especies), vulnerables (6 especies) y casi amenazadas (7 especies) (Tabla 3).

Tabla 3. Especies de plantas medicinales amenazadas

\begin{tabular}{|c|c|c|c|}
\hline Especies & $\begin{array}{l}\text { Nombre común en } \\
\text { el Perú }\end{array}$ & $\begin{array}{c}\text { Parte } \\
\text { comercializada }\end{array}$ & $\begin{array}{c}\text { Categoría } \\
\text { de amenaza }\end{array}$ \\
\hline Acaulimalva engleriana (Ulbr.) Krapov. & Altea & Planta entera & NT \\
\hline Baccharis genistelloides (Lam.) Pers. & Carqueja & Parte aérea & NT \\
\hline Mutisia acuminata Ruiz \& Pav. & Chinchilcoma, chinchilcuma & Parte aérea & NT \\
\hline Gentianella thyrsoidea (Hook.) Fabris & Chinchimali, corpus huay & Planta entera & VU \\
\hline Argyrochosma nivea (Poir.) Windham & Cuti cuti hembra & Planta entera & VU \\
\hline Buddleja incana Ruiz \& Pav. & Flor blanca & Parte aérea & CR \\
\hline Chuquiraga spinosa Less. & Huamampinta & Parte aérea & NT \\
\hline Senecio rhizomatus Rusby & Llancahuasha & Planta entera & VU \\
\hline Desmodium molliculum (Kunth) DC. & Manayupa & Parte aérea & NT \\
\hline Juglans neotropica Diels & Nogal & Hojas & NT \\
\hline Geranium sp. & Pasuchaca & Planta entera & EN \\
\hline Polylepis racemosa Ruiz \& Pav. & Quinual & Parte aérea & $\mathrm{CR}$ \\
\hline Krameria lappacea (Dombey) Burdet \& B.B. Simpson & Ratania & Planta entera & EN \\
\hline Laccopetalum giganteum (Wedd.) Ulbr. & Rima rima & Flores & CR \\
\hline Corryocactus brevistylus (K.Schum. ex Vaupel) Britton \& Rose & Sanky & Frutas & VU \\
\hline Caesalpinia spinosa (Molina) Kuntze & Tara & Frutas & VU \\
\hline Senecio nivalis (Kunth) Cuatrec. & Wiña, wiña wiña & Planta entera & VU \\
\hline Eleutherine bulbosa (Miller.) Urban. & Yahuar piri piri & Planta entera & NT \\
\hline
\end{tabular}

$\mathrm{CR}=$ En peligro crítico, $\mathrm{EN}=$ En peligro, $\mathrm{VU}=$ Vulnerable, $\mathrm{NT}=$ Casi amenazado.

\section{DISCUSIÓN}

Estudios etnobotánicos realizados en mercados locales, muestran una relación entre la diversidad de plantas medicinales y el área geográfica ${ }^{(9,16)}$, como evidencian los patrones encontrados en Cusco, Cochabamba y $\mathrm{La} \mathrm{Paz}{ }^{10,16}$ 18). Así también, los mercados de Lima, que representan a un conjunto de culturas de todo el país, tienen una cantidad de especies similares a las reportadas en Colombia ${ }^{(12)}$ y superiores a las registradas en Brasil ${ }^{(11,19)}$, Ecuador ${ }^{(6)}$, Bolivia ${ }^{(18)}$, México ${ }^{(8)}$ y Venezuela ${ }^{(20)}$.

En el Perú, las familias botánicas más representativas, en términos de cantidad, fueron Asteraceae (20,2 \%) y Lamiaceae $(9,3 \%)$, como ya se ha observado en otros países ${ }^{(17,18)}$. Sin embargo, las Fabaceae $(6,6 \%$ ) no habían sido reportadas anteriormente en nuestro medio, aunque en algunos países sudamericanos predominan las tres familias Asteraceae ${ }^{(6,16,21)}$, Lamiaceae ${ }^{(16)}$ y Fabaceae ${ }^{(18)}$.

En la comercialización de plantas medicinales existen, sin duda, elementos conceptuales asociados a la interacción de la medicina tradicional y la medicina oficial, donde la información sobre la validación de la primera se encuentra totalmente dispersa ${ }^{(5)}$. Por otro lado, estos especímenes se venden de manera informal como "atados" y "paquetes", 
lo que dificulta calcular el volúmen de venta; esta situación se repite en otros países de la región $(5,8,16,21)$. En los mercados de Lima se comercializan las plantas, sus partes o mezclas de estas, las mismas que se compran y venden por su nombre común, por lo que el usuario no puede verificar su autenticidad (22). Asimismo, las condiciones sanitarias afectan la calidad de las medicinas naturales $y$, en muchos casos, el comerciante sobredimensiona sus beneficios y no se advierte al usuario sobre posibles efectos adversos o contraindicaciones. Este hallazgo es consistente con otros estudios ${ }^{(23,24)}$ que muestran que existe una gran variedad de especies identificadas con el mismo nombre común, varios nombres comunes para una especie, así como inconsistencias en relación con la dosis, el uso y los efectos secundarios.

Se observó un predominio de las especies nativas $(66,1 \%)$, similar, pero en menor proporción a lo registrado en Brasil (2), Ecuador ${ }^{(6,25)}$ y Bolivia ${ }^{(17)}$, que podría evidenciar la desvalorización, depredación o, como algunos autores afirman, la influencia de la conquista española en la introducción de plantas medicinales a nuestras culturas (6). Además, el mayor número de especies silvestres registradas en los mercados populares de Lima, motiva la necesidad de establecer políticas de conservación alineadas con las directrices para la identificación, conservación y uso sostenible de plantas medicinales, impartido por la Organización Mundial de la Salud (OMS) ${ }^{(26)}$ y la organización TRAFFIC (27). Además, esta investigación confirma, como otras (28), que muchas de las plantas medicinales que se venden, se encuentran en peligro o amenaza, lo que evidencia el impacto del comercio sobre las poblaciones de flora silvestre.

Muchos estudios ${ }^{(3,29,30)}$ han demostrado la gran diversidad de conocimientos sobre las plantas medicinales por parte de los practicantes de la medicina tradicional; sin embargo, la evaluación bioprospectiva de plantas medicinales y usos tradicionales también se puede determinar en los mercados locales y revelar sus riesgos y oportunidades, tanto para la salud como para el medio ambiente. La gran demanda de estos productos confirma su valor como recurso terapéutico y la importancia que tienen para las personas en el tratamiento de enfermedades; sin embargo, se requiere un mayor control sobre su venta para garantizar la salud pública y su disponibilidad a lo largo del tiempo. Durante el año, la oferta se modifica en función de la demanda. Los productos utilizados para el tratamiento de enfermedades respiratorias $y$ afecciones del sistema musculoesquelético (articulaciones) aumentan su demanda durante los meses de invierno; mientras que los empleados para la prevención o tratamiento de trastornos gastrointestinales y problemas hepáticos se mantienen durante todo el año; este panorama difiere de lo reportado en el interior del país, donde el número de especies varía según las estaciones de lluvia y sequía (10). Por lo general, las plantas se utilizan en primer lugar, para el tratamiento de trastornos gastrointestinales y enfermedades del hígado, seguidas de enfermedades respiratorias, sistema genitourinario, alteraciones del sistema circulatorio y sistema musculoesquelético; estas características no concuerdan con otros estudios realizados en localidades como Pastaza, en la amazonía ecuatoriana ${ }^{(31)}$.

Aunque nuestra investigación no tiene representatividad a nivel nacional, muestra el riesgo de la población a una serie de problemas de salud, así como los problemas futuros de sostenibilidad de las plantas medicinales, lo que genera una serie de preguntas que se pueden investigar en el futuro inmediato.

En conclusión, las plantas medicinales vendidas en los mercados evaluados provienen, principalmente, de las tierras altas peruanas, y se presentan, sobre todo, en "atados". La mayoría de ellas son nativas, se comercializan frescas y se ofrecen para el tratamiento de varios problemas de salud. En invierno, aumenta la demanda a más de 85 especies, de las cuales, la manzanilla, el orégano, el cedrón, el toronjil, la yerbaluisa, el hinojo, la muña, la sábila o aloe, el llantén y la coca son las más solicitadas. Se destaca que, del total de especies comercializadas, el $10 \%$ está en peligro de extinción. Por todo esto, nuestro estudio revela la importancia de generar iniciativas para el desarrollo de investigaciones e intervenciones en este campo, para lograr la formalización y un mayor control en la venta de estos productos.

\section{REFERENCIAS BIBLIOGRÁFICAS}

1. Morón Rodríguez FJ. La biodiversidad y las plantas medicinales en riesgo. Rev Cubana Plant Med. 2008; 13(3).

2. Ribeiro Silva S, Buitrón X, De Oliveira LH, Martins MVM. Plantas medicinales de Brasil: aspectos generales sobre legislación y comercio. 2001.

3. Sen T, Samanta SK. Medicinal plants, human health and biodiversity: a broad review. Adv Biochem Eng Biotechnol. 2014; 147: 59-110.

4. Brandão Md, Cosenza GP, Pereira FL, Vasconcelos AS, Fagg CW. Changes in the trade in native medicinal plants in Brazilian public markets. Environ Monit Assess. 2013; 185(8): 7013-23.

5. Acosta ME, Ladio AH, Vignale ND. Plantas medicinales comercializadas en la ciudad de San Salvador de Jujuy (Argentina) y su calidad botánica. Bol Latinoam Caribe Plant Med Aromat. 2017; 16(1): 34-52.

6. Cerón Martínez CE. Plantas medicinales de los andes ecuatorianos. Botánica Económica los Andes Cent. 2006; 28593.

7. Leitão F, Leitão SG, De Almeida MZ, Cantos J, Coelho T, Da Silva PEA. Medicinal plants from open-air markets in the State of Rio de Janeiro, Brazil as a potential source of new antimycobacterial agents. J Ethnopharmacol. 2013; 149(2): 513-21.

8. Aldama Meza JNL, Rodríguez Ramos IE, Gómez Peña JJ, 
Orozpe Olvera JA, Carbajal De la Rosa ND. Caracterización del comercio de plantas medicinales en ciudad Juárez, Chihuahua, México. Cult Cient Tecnol. 2015; 12(57): 331-41.

9. Ortega FJ. Variación en el conocimiento de uso de la flora medicinal en las veredas de Chaves, Guadalupe y Alto Casanare con relación al Centro Urbano del corregimiento de Catambuco, Municipio de Pasto, Sur de Colombia. Universidad de Nariño; 2014.

10. Huamantupa I, Cuba M, Urrunaga R, Paz E, Ananya N, Callalli $M$, et al. Riqueza, uso y origen de plantas medicinales expendidas en los mercados de la ciudad del Cusco. Rev Peru Biol. 2011; 18(3): 283-91.

11. Monteiro JM, Araújo EL, Cavalcanti Amorim EL, De Alburquerque UP. Local markets and medicinal plant commerce. A review with emphasis on Brazil. Econ Bot. 2010; 64(4): 352-66.

12. Rivera A, Buitrón X, Rodríguez P. Uso y comercio sostenible de plantas medicinales en Colombia. Rivera A, Buitrón $X$, Rodríguez P, editors. Quito: TRAFFIC América del Sur; 2000. $\mathrm{p}$ 88.

13. Brako L, Zarucchi JL. Catalogue of the flowering plants and gymnosperms of Peru (monographs in systematic botany fro). Mo Bot Gard; 1993. p 1286.

14. Ministerio de Agricultura. Aprueban categorización de especies amenazadas de flora silvestre. Decreto Supremo No 043-2006-AG de 7 de julio Perú: Diario Oficial El Peruano, N ${ }^{\circ}$ 9526 (13-07-2006). p 323527-39.

15. The Angiosperm Phylogeny Group. An update of the Angiosperm Phylogeny Group classification for the orders and families of flowering plants: APG III. Bot J Linn Soc. 2009; 161(2): 105-21.

16. Bussmann RW, Paniagua Zambrana NY, Moya Huanca LA, Hart R. Changing markets - medicinal plants in the markets of La Paz and El Alto, Bolivia. J Ethnopharmacol. 2016; 193: 76-95.

17. Justo-Chipana $M$, Moraes $M$. Plantas medicinales comercializadas por las chifleras de La Paz y El Alto (Bolivia). Ecol en Boliv. 2015; 50(2): 66-90.

18. Macía MJ, García E, Vidaurre PJ. An ethnobotanical survey of medicinal plants commercialized in the markets of la Paz and El Alto, Bolivia. J Ethnopharmacol. 2005; 97(2): 337-50.

19. Bieski IG, Leonti M, Arnason JT, Ferrier J, Rapinski M, Violante $I M$, et al. Ethnobotanical study of medicinal plants by population of Valley of Juruena Region, Legal Amazon, Mato Grosso, Brazil. J Ethnopharmacol. 2015; 173: 383-423.

20. Giraldo D, Baquero E, Bermúdez A, Oliveira-Miranda T. Caracterización del comercio de plantas medicinales en los mercados populares de Caracas, Venezuela. Acta Bot Venez. 2009; 32(2): 267-301.

21. Puelles Gallo M, Gómez Galarza V, Gabriel y Galán Moris JM, De Felipe Boente I, Briz Escribano J, Siura Céspedes S, et al. Las plantas medicinales de Perú: etnobotánica y viabilidad comercial. Madrid: Catarata; 2010. p 144.

22. Costa Lima PG, Coelho-Ferreira M, Da Silva Santos R. Perspectives on medicinal plants in public markets across the amazon: a review. Econ Bot. 2016; 70(1): 64-78.

23. Bussmann RW, Paniagua-Zambrana N, Chamorro MR, Molina Moreira N, Del Rosario Cuadros Negri ML, Olivera J. Peril in the market-classification and dosage of species used as antidiabetics in Lima, Peru. J Ethnobiol Ethnomed. 2013; 9: 37.

24. Giraldo Quintero SE, Bernal Lizarazú MC, Morales Robayo

A, Pardo Lobo AZ, Gamba Molano L. Descripción del uso tradicional de plantas medicinales en mercados populares de Bogotá, D. C. Nova. 2015; 13(23): 73-80.

25. Tinitana F, Rios M, Romero-Benavides JC, De La Cruz Rot M, Pardo-de-Santayana M. Medicinal plants sold at traditional markets in southern Ecuador. J Ethnobiol Ethnomed. 2016; 12(1): 1-18.

26. Organización Mundial de la Salud. Estrategia de la OMS sobre medicina tradicional 2014-2023. Hong Kong SAR, China; 2013. $\mathrm{p} 72$.

27. TRAFFIC. Programme summary. Working to ensure that trade in wild animals and plants is not a threat to the conservation of nature. Cambridge; 2016.

28. Abbasi AM, Khan MA, Shah MH, Shah MM, Pervez A, Ahmad M. Ethnobotanical appraisal and cultural values of medicinally important wild edible vegetables of Lesser HimalayasPakistan. J Ethnobiol Ethnomed. 2013; 9(1): 66-78.

29. Balamurugan S, Vijayakumar S, Prabhu S, Morvin Yabesh JE. Traditional plants used for the treatment of gynaecological disorders in Vedaranyam taluk, South India - an ethnomedicinal survey. J Tradit Complement Med. 2017; 8(2): 308-23.

30. Bruschi P, Mancini M, Mattioli E, Morganti M, Signorini MA. Traditional uses of plants in a rural community of Mozambique and possible links with miombo degradation and harvesting sustainability. J Ethnobiol Ethnomed. 2014; 10: 59-80.

31. Saltos RVA, Vásquez TER, Lazo JA, Banguera DV, Guayasamín PDR, Vargas JKA, et al. The use of medicinal plants by rural populations of the Pastaza province in the Ecuadorian Amazon. Acta Amazon. 2016; 46(4): 355-66.

Fuentes de financiamiento:

Este artículo ha sido financiado por los autores.

Conflicto de interés:

Los autores declaran no tener ningún conflicto de interés.

\section{Correspondencia:}

Jesús Tomás Silva Alarcón

Dirección: Av. Defensores del Morro 2268 (Ex Huaylas) - Chorrillos. Lima, Perú.

Telefono: 7480000 Anexo 1203

Correo electronico: jesusicon1@gmail.com

\section{Recibido: 03 de diciembre de 2018. Evaluado: 31 de enero de 2019 Aprobado: 21 de febrero de 2019.}

(c) La revista. Publicado por Universidad de San Martín de Porres, Perú. (c) $\mathbf{\text { Br }}$ Licencia de Creative Commons Artículo en acceso abierto bajo términos de Licencia Creative Commons Atribución 4.0 Internacional. (http://creativecommons.org/licenses/by/4.0/)

\section{ORCID iDs}

Jesús Silva Alarcón

Jorge Cabrera Meléndez

Omar V. Trujillo Villarroel

Ivonne F. Reyes-Mandujano https://orcid.org/0000-0002-7676-0490 https://orcid.org/0000-0002-1592-8325 https://orcid.org/0000-0002-1431-0427 https://orcid.org/0000-0001-7588-3758 